\title{
Search for Higgs boson Decays to Beyond-the-Standard-Model Light Bosons in Four-Lepton Final States with the ATLAS Detector at the LHC
}

\author{
Zainab Soumaimi, ${ }^{1}$ Diallo Boye, ${ }^{2}$ Farida Fassi, ${ }^{1}$ Ketevi Assamagan, ${ }^{3}$ Simon Connell, ${ }^{2}$ and Christian Weber ${ }^{3}$ \\ ${ }^{1}$ Mohammed V University in Rabat, Morocco \\ ${ }^{2}$ University of Johannesburg, South Africa \\ ${ }^{3}$ Brookhaven National laboratory, USA
}

\begin{abstract}
Hidden sector or dark sector states appear in many extensions to the Standard Model (SM), to provide particle mediators for dark matter in the universe. A new probe of this hypothetical hidden or dark sector may have become available at the energy frontier opened up by the LHC with the Higgs boson and its distinct couplings to SM particles. A search is conducted for a beyond-the-Standard-Model vector boson using events where a Higgs boson with mass $125 \mathrm{GeV}$ decays to four leptons. This decay is presumed to occur via an intermediate state which contains one or two decaying light exotic bosons, $H \rightarrow Z\left(Z_{d}\right) Z_{d} \rightarrow$ $4 l(l=e, \mu)$, where $Z_{d}$ is a new vector boson with mass between 1 and $60 \mathrm{GeV}$. The search uses pp collision data collected with the ATLAS detector at the LHC with an integrated luminosity of $36 \mathrm{fb}^{-1}$ at the centerof-mass energy of $13 \mathrm{TeV}$. No significant excess of events above SM background predictions is observed; therefore, upper bounds on the branching ratios $\mathrm{BR}\left(H \rightarrow Z_{d} Z_{d} \rightarrow 4 l\right)$ are set as a function of the mass of the dark vector boson at $95 \%$ confidence level.
\end{abstract}

Keywords: exotic bosons, hidden sector, $U(1) d$ symmetry, kinetic an Higgs mixing parameters,

standard model extension, upper limits

DOI: $10.31526 /$ ACP.BSM-2021.7

\section{INTRODUCTION}

For a better understanding of the dark force particles, a set of models, where the Standard Model (SM) is extended by introducing hidden or dark sector states, is adopted in a way that provides candidates for dark matter and dark forces which accommodate both the indirect and the (potential) direct evidence based on astronomical observations or space platform experiments. In this case, the dark sector is introduced with an additional $U(1)_{d}$ dark gauge symmetry $[1,2,3,4,5,6,7,8,11,12]$.

In this analysis, a Higgs Portal model, which has a Higgs level coupling between the dark sector and the SM, is considered. Accordingly, the $U(1)_{d}$ symmetry is broken by the introduction of a dark Higgs boson, which mixes with the SM Higgs boson with a coupling strength $\kappa$.

The observed Higgs boson would then be the lighter partner of the new Higgs doublet, which can also decay via the dark sector. The dark sector can additionally couple to the SM through kinetic mixing with the hypercharge gauge via the kinetic mixing parameter $\epsilon$ to allow the decay $Z_{d} \rightarrow l l$. The current EWPO restrict the hypercharge portal to a greater degree than the Higgs Portal. We can further assume the dark fermions are sufficiently heavy $m_{f_{d}}<m_{Z_{d}} / 2$, so that the branching ratio for the decay $Z_{d} \rightarrow l l$ may be taken as $100 \%$, even though the kinetic mixing parameter $\epsilon$ can be set small to be $\epsilon \approx 10^{-4}$, and still satisfy the requirement for prompt decays.

In this proceeding article, the Run2 results, for the Higgs boson decaying to four leptons by the intermediate of two $Z_{d}$ bosons, are discussed using pp collision data at $\sqrt{s}=13 \mathrm{TeV}$ for an integrated luminosity of $36 \pm 1.2 \mathrm{fb}^{-1}$ collected with the ATLAS detector at LHC.

The decays of $Z_{d}$ bosons to same-flavor particles (electron and muon pairs) are considered in this search, producing $4 \mathrm{e}, 2 \mathrm{e} 2 \mu$ and $4 \mu$ in the final state. Cases where the $\tau$ leptons are produced, are not taken in consideration.

Since no significant excess of events above SM background predictions is observed, a 95\% CL upper limit was set on the modelindependent fiducial cross section for the process $H \rightarrow Z_{d} Z_{d} \rightarrow 4 l$ as well as considering the benchmark model where the SM was extended by an additional $U(1)_{d}$ gauge symmetry, upper limits could be set on the branching ratio $\mathcal{B R}\left(H \rightarrow Z_{d} Z_{d}\right)$.

In this paper, the search is conducted for a Higgs boson with $m_{H}=125 \mathrm{GeV}$ decaying to an on-shell $Z_{d}$ boson in a mass range of $15<m_{Z_{d}}<60 \mathrm{GeV}$.

\section{EXPERIMENTAL SETUP: SIGNAL AND BACKGROUNDS SIMULATION}

The ATLAS detector covers almost the whole solid angle around the collision point with layers of tracking detectors, calorimeters and muon chambers. Further details can be found in [9]. Event preselection, trigger and lepton reconstruction procedure are as 
detailed in [10]. The quadruplets are then formed by these leptons according to the selection criteria cited in the table below.

\begin{tabular}{l|l}
\hline Object & $H \rightarrow Z_{d} Z_{d} \rightarrow 4 l$ \\
\hline \hline & - In each event, a quadrulpet is formed from two lepton pairs each with same flavour \\
& opposite sign leptons: "1,2" and "3,4". \\
Quadruplet selection & - Each lepton should fire at least 1 trigger. \\
& - Three leading-pt leptons must have: $p t>20,15$ and $10 \mathrm{GeV}$. \\
& $-\Delta R\left(l, l^{\prime}\right)>0.10(0.20)$ for same-flavour (different-flavour) leptons in the quadruplet. \\
\hline \multirow{2}{*}{ Quadruplet ranking } & - The selected quadruplet should have the smallest difference in mass between lepton pairs: \\
& $\Delta m_{l l}=\left|m_{12}-m_{34}\right|$ \\
\hline \multirow{5}{*}{ Event selection } & - Higgs boson mass window:115 GeV $<m_{4 l}<130 \mathrm{GeV}$ \\
& - Z veto: $10 \mathrm{GeV}<m_{12,34}<64 \mathrm{GeV}$ and $5 \mathrm{GeV}<m_{14,32}<75 \mathrm{GeV}$ \\
& - Quarkonia veto: event is rejected if either $($ or both) condition are fulfilled \\
& $\left(m_{J / \Psi}-0.25 \mathrm{GeV}\right)<m_{12,34,14,32}<\left(m_{\Psi}(2 S)+0.30 \mathrm{GeV}\right)$ or \\
& $\left(m_{\mathrm{Y}(1 S)}-0.70 \mathrm{GeV}\right)<m_{12,34,14,32}<\left(m_{\mathrm{Y}(3 S)}+0.75 \mathrm{GeV}\right)$ \\
\hline & $-m_{34} / m_{12}>0.85$ \\
\hline \hline
\end{tabular}

TABLE 1: Summary of the event selection

Simulated event samples are used to model the signal process, and to estimate most of the SM backgrounds.

\subsection{Signal}

The $H \rightarrow Z_{d} Z_{d} \rightarrow 4 l$ signal is generated by configuring the event generator with the Hidden Abelian Higgs Model (HAHM) $[11,12]$. The event generator MADGRAPH5 [13], at next-to-leading order (NLO) with CTEQ6L1 parton distribution functions (PDFs) [14], is interfaced with PYTHIA8(v8.170) for the modelling of the parton shower, hadronisation and underlying event(UE).

The $\mathrm{Zd}$ boson mass is varied for different signal hypotheses in the range of $15 \mathrm{GeV}<m_{Z_{d}}<60 \mathrm{GeV}$ for $H \rightarrow Z_{d} Z_{d} \rightarrow 4 l$, in 5 $\mathrm{GeV}$ steps. The Higgs boson $\left(m_{H}=125 \mathrm{GeV}\right)$ production in the gluon fusion (ggF) mode.

\subsection{Backgrounds}

$H \rightarrow Z^{*} \rightarrow 4 l:$

The event generator PowHEG-Box v2 [15] is considered to simulate Higgs production through ggF, vector-boson fusion (VBF) and in association with a vector-boson $(\mathrm{VH})$ processes, using the PDF4LHC NLO PDF set. The processes with Higgs boson production in association with a heavy quark pair are simulated with MADGRAPH5_aMC@NLO [16] using CT10nlo PDF set [17] and NNPDF23 PDF set [18] for $t \bar{t} H$ and $b \bar{b} H$ respectively. The modeling of parton showering, hadronisation, and multiple partonparton interactions for ggF, VBF, VH, and $b \bar{b} H$ production mechanisms is done by PYTHIA8 using the AZNLO parameter set [19]. For $t \bar{t} H$ process showering, the event generator HERWIG++ [20] is considered with usage of UEEE5 parameter set [21].

$\mathrm{ZZ}^{*} \rightarrow 4 l:$

This process is dominated by $q \bar{q}$ production mechanism and it is simulated by POWHEG interfaced to PYTHIA8. The modeling of $\mathrm{ggF}, \mathrm{VBF}, \mathrm{VH}, t \bar{t} H$ and $b \bar{b} H$ processes is the same adopted in $H \rightarrow Z Z^{*} \rightarrow 4 l$ simulation.

EWK $6 \rightarrow 4 l+2 X:$

Higher-order electroweak processes (with cross sections proportional to $\alpha^{6}$ at leading order) include triboson production(VVV) and vector-boson scattering(VBS), which lead to four leptons in the final state, with two additional particles (quarks, neutrinos, or electrons and muons). These processes are modelled by SHERPA 2.1 with the CT10 PDFs.

$Z+(t \bar{t} / J / \psi / Y) \rightarrow 4 l:$

This background process corresponds to the production of a $Z$ boson, in association with either a quarkonium state $(\boldsymbol{b} \bar{b}$ or $c \bar{c})$ that decays to leptons, or $t \bar{t}$ production with leptonic decays of the prompt $\mathrm{W}$ bosons. The ttZ process is modelled at leading order with MADGRAPH5 interfaced to PYTHIA8, using the NNPDF23 PDFs and the A14 tune [22].

\section{Reducible background:}

Precesses producing less than four prompt leptons, like $Z+j e t s, t \bar{t}$ and $\mathrm{WZ}$, are also contributing to the selection through jets misidentified as leptons. The $Z+$ jets events are modeled using SHERPA 2.2 while $t \bar{t}$ is generated with POWHEG interfaced to PYTHIA6. The WZ production is modelled by POWHEG interfaced to PYTHIA8 and the CT10 PDFs. 


\section{ANALYSIS PROCEDURE}

\section{Backgrounds contribution:}

The background processes that are mainly contributing to this analysis are $H \rightarrow Z Z^{*} \rightarrow 4 l$ and $Z Z^{*} \rightarrow 4 l$ with $63 \%$ and $19 \%$ of the total prediction, respectively. The Higher-order electroweak processes(VVV, VBS), which lead to four leptons in the final state, with two additional particles, are contributing with a smaller portion of $17 \%$ of the total prediction. While the production of $Z+(t \bar{t} / J / \psi / Y) \rightarrow 4 l$ and $Z+t \bar{t} \rightarrow 4 l$ constitute another minor source of background, accounting for approximately $1 \%$ of the total.

All the background processes mentioned above are estimated using MC simulation and normalised to the theoretical calculations of their cross sections.

\section{Systematics:}

The main origin of systematic uncertainties which affect the normalisation or the shape of the signal and background samples is the imperfect knowledge of the parameters affecting the measurements either from simulated or from data- driven estimates. The well recognition of these uncertainties leads to a better identification of the significance when talking about a discovery and the limit when talking about exclusion. Further details can be found in [10].

\section{RESULTS}

The $\left\langle m_{l l}\right\rangle=\frac{1}{2}\left(m_{12}+m_{34}\right)$ distributions for the events selected in this analysis are shown in figure 1. Six events are observed for a prediction of $3.9 \pm 0.3$ events in the high-mass selection.

The biggest deviation from the SM expectation is from a single event at $\left\langle m_{l l}\right\rangle \approx 20 \mathrm{GeV}$, with a local significance of $3.2 \sigma$ in the $2 \mathrm{e} 2 \mu$ channel. The corresponding global significance is approximately $1.9 \sigma$, estimated using an approximation for the tail probability of the profile-likelihood-ratio test statistic [23].

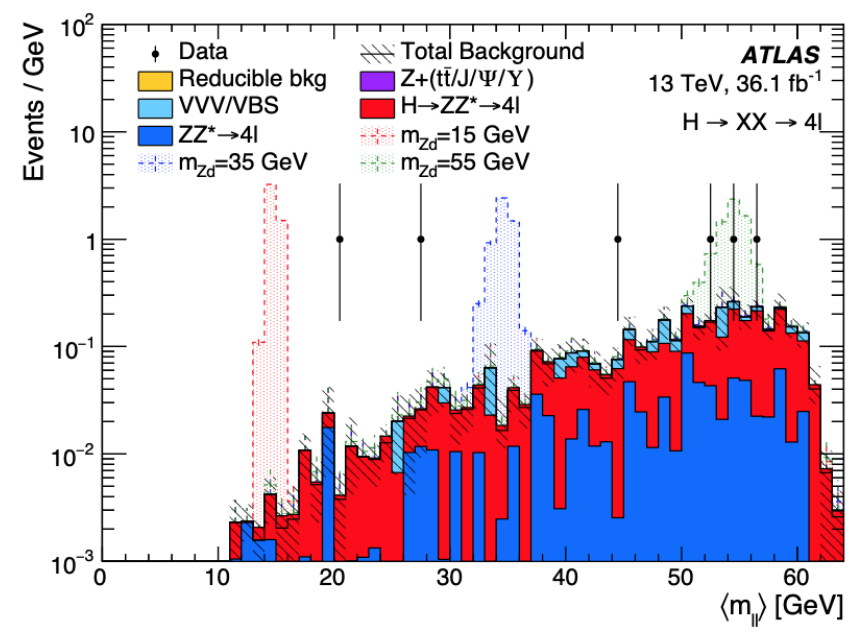

FIGURE 1: Distribution of the dilepton mass average $\left\langle m_{l l}\right\rangle$ in Signal region with all channels combined in 2015-6 data.

The results do not statistically show any evidence for the signal processes of $H \rightarrow Z_{d} Z_{d} \rightarrow 4 l$, so they are therefore interpreted in terms of limits on the benchmark models [7, 8].

A fiducial phase space was chosen in a way to mimic the analysis selections described above to compute the reconstruction efficiencies. These efficiencies are used to compute 95\% CL upper limits on the cross sections in the fiducial phase space using the CLs frequentist formalism with the profile-likelihood test statistic [24]. The results are shown at the right in figure 2.

Model-dependent acceptances for the fiducial phase spaces are computed per channel for the $H \rightarrow Z_{d} Z_{d} \rightarrow 4 l$ search. The acceptances are used to compute upper limits on $\sigma_{H} \cdot \mathcal{B R}\left(H \rightarrow Z_{d} Z_{d} \rightarrow 4 l\right)$ and these cross section limits are converted into limits on the branching ratios of $\boldsymbol{H} \rightarrow Z_{d} Z_{d}$ by using the theoretical branching ratios $Z_{d} \rightarrow l l$ from the benchmark model. The limits on these branching ratios are shown at the left in figure 2 for the $H \rightarrow Z_{d} Z_{d} \rightarrow 4 l$ search.

The branching ratio limit can also be interpreted as a limit on the Higgs mixing parameter $\kappa$ :

$$
\kappa^{\prime}=\kappa \times \frac{m_{H}^{2}}{\left|m_{H}^{2}-m_{S}^{2}\right|},
$$

where $\kappa$ is the Higgs portal coupling and $m_{S}$ is the mass of the dark Higgs boson. The limits on the higgs mixing parameter are shown in figure 3 . 

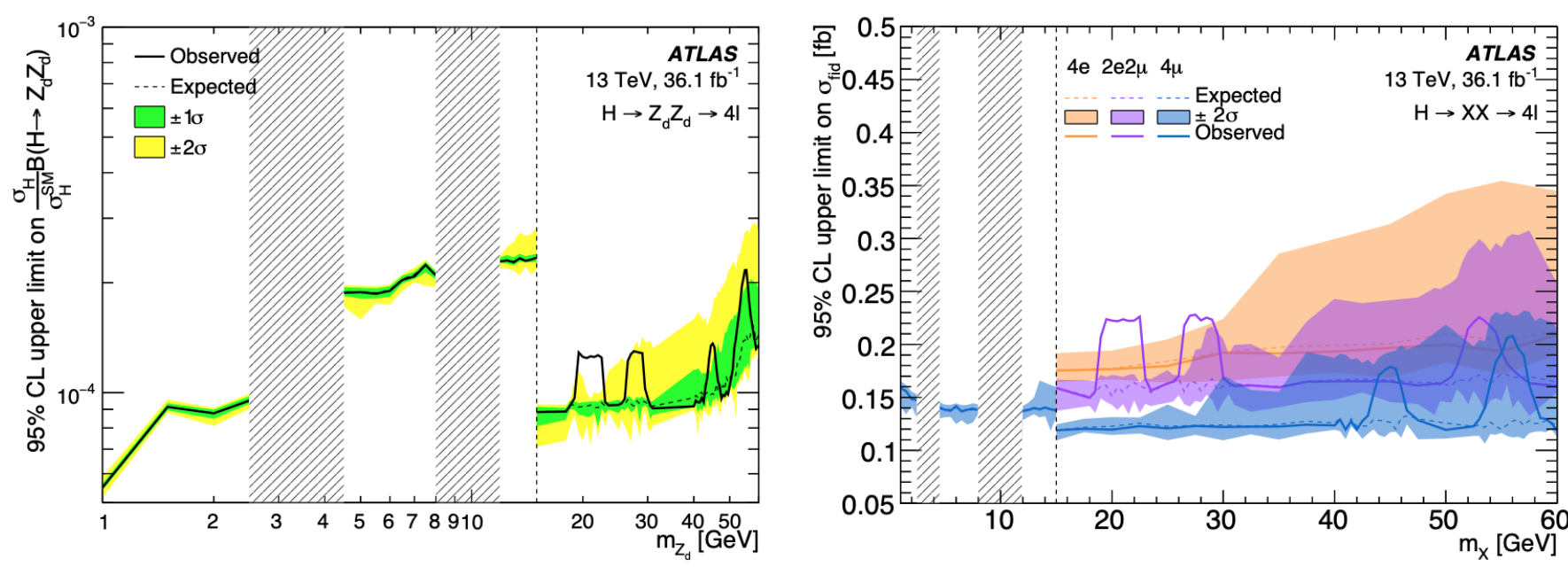

FIGURE 2: $95 \%$ CL upper limits of the process $H \rightarrow X X \rightarrow 4 l$ on (right) model-independent fiducial cross sections, and (left) branching fractions $\mathcal{B} \mathcal{R}\left(H \rightarrow Z_{d} Z_{d}\right)$ for the benchmark model.

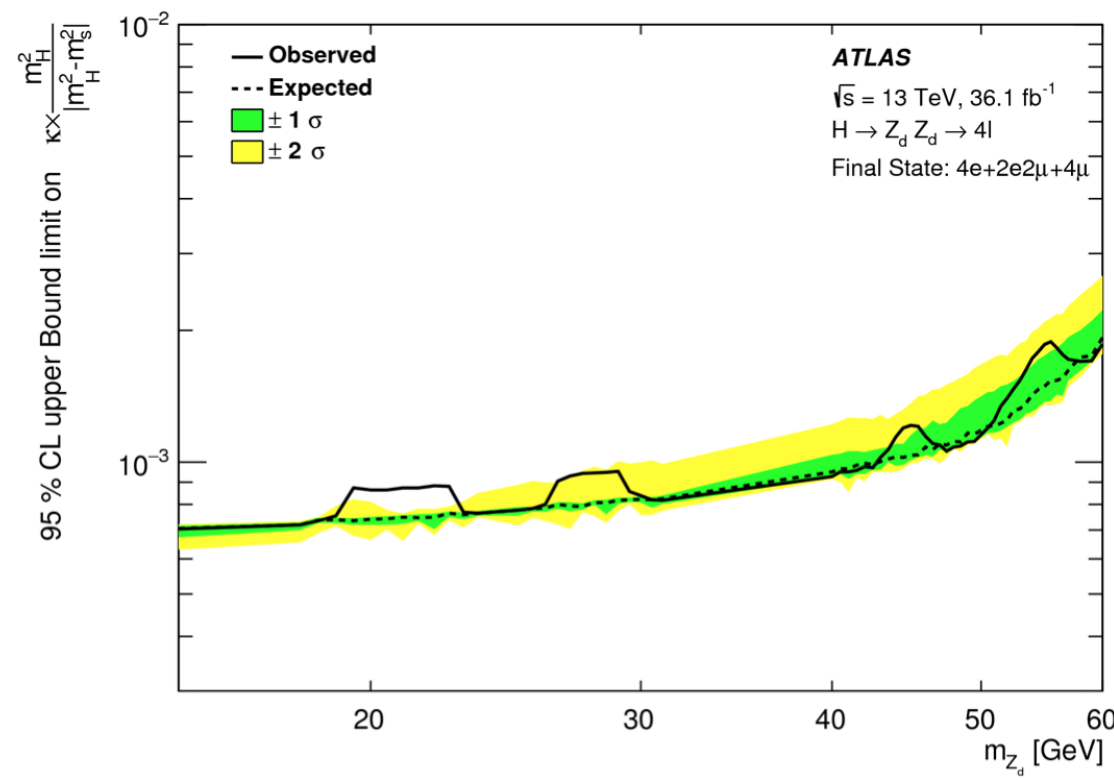

FIGURE 3: Upper limit at 95\% CL on the effective Higgs mixing parameter $\boldsymbol{\kappa}^{\prime}$.

\section{CONCLUSION}

A search for an exotic gauge boson $\mathrm{Zd}$ that couples to the discovered SM Higgs boson at a mass around $125 \mathrm{GeV}$ in four-lepton events is presented in the present proceeding paper. Six events are observed for a background prediction of 3.9 \pm 3 . The results are expressed in terms of upper limits on the branching ratios $\mathcal{B R}\left(H \rightarrow Z_{d} Z_{d}\right)$ as a function of $m_{Z_{d}}$ for the HAHM (Hidden Abelian Higgs Model) benchmark model as wall as on the Higgs mixing parameter $\kappa$. Limits are also provided on model-independent fiducial cross sections.

\section{References}

[1] Fayet P 2004 Phys.Rev. D 70023514 (Preprint hep-ph/0403226)

[2] Finkbeiner D P and Weiner N 2007 Phys.Rev. D 76083519 (Preprint astro-ph/0702587)

[3] Arkani-Hamed, N et al 2009 Phys.Rev. D 79015014 (Preprint 0810.0713)

[4] Dudas, E et al 2012 J. High Energy Phys. 1210123 (Preprint 1205.1520)

[5] Davoudiasl H, Lee H S, Lewis I and Marciano W J 2013 Phys.Rev. D 88015022 (Preprint 1304.4935)

[6] Davoudiasl H, Lee H S and Marciano W J 2012 Phys.Rev. D 85115019 (Preprint 1203.2947)

[7] Curtin D, Essig R, Gori S and Shelton J 2015 J. High Energy Phys. 1502157 (Preprint 1412.0018)

[8] Curtin D et al. 2014 Phys.Rev. D 90075004 (Preprint 1312.4992)

[9] ATLAS Collaboration 2009 (Preprint 0901.0512)

[10] ATLAS Collaboration 2018 J. High Energy Phys. 1806166 (Preprint 1802.03388)

[11] Wells J D 2008 (Preprint 0803.1243) 
[12] Gopalakrishna S, Jung S and Wells J D 2008 Phys.Rev. D 78055002 (Preprint 0801.3456)

[13] Alwall, J et al 2011 J. High Energy Phys. 1106128 (Preprint 1106.0522)

[14] Pumpkin, J et al 2002 JHEP 07012 (Preprint hep-ph/0201195)

[15] Alioli S, Nason P, Oleari C and Re E 2010 JHEP 06043 (Preprint 1002.2581)

[16] Alwall, J et al 2014 JHEP 07079 (Preprint 1405.0301)

[17] Lai, H-L et al 2010 Phys. Rev. D82 074024 (Preprint 1007.2241)

[18] Ball RD et al, (NNPDF Collaboration) 2013 Nucl. Phys. B 867 244-289

[19] ATLAS Collaboration 2014 JHEP 09145 (Preprint 1406.3660)

[20] Bahr M et al. 2008 Eur. Phys. J. C58 639-707 (Preprint 0803.0883)

[21] Seymour M H and Siodmok A 2013 JHEP 10113 (Preprint 1307.5015)

[22] ATLAS Collaboration 2014 ATL-PHYS-PUB-2014-021 URL http://cdsweb.cern.ch/record/1966419

[23] Gross E and Vitells O 2010 Eur. Phys. J. C70 525-530 (Preprint 1005.1891)

[24] Read A L 2002 Journal of Physics G 282693 URL http:/ / stacks.iop.org/0954-3899/28/i=10/a=313 